\title{
Stability Analysis and Design of Negative Impedance Converters: Application to Circuit and Small Antennas
}

\author{
Daniel SEGOVIA-VARGAS ${ }^{1}$, José Luis JIMÉNEZ-MARTÍN ${ }^{2}$, Ángel PARRA-CERRADA ${ }^{2}$, \\ Fernando ALBARRACIN-VARGAS ${ }^{1}$, Eduardo UGARTE-MUNOOZ ${ }^{1}$, Vicente GONZALEZ-POSADAS ${ }^{2}$ \\ ${ }^{1}$ Dept. of Signal Theory and Comm., Carlos III University of Madrid, Av. La Universidad 30, 28911, Leganés, Spain \\ ${ }^{2}$ Dept. of Audiovisuals and Comm., Polytechnic University of Madrid, Km 7 Valencia-Highway, 28031 Madrid, Spain
}

dani@tsc.uc3m.es, lalbarra@ing.uc3m.es, vgonzalz@diac.upm.es

Manuscript received June 2, 2016

\begin{abstract}
Negative impedance converters (NICs) have been proposed as structures to improve the performance of $R F$ circuits and electrically small antennas. However, NICs suffer from stability problems. This paper presents a compact procedure to analyze the stability of NICs. Then, the required and sufficient conditions to predict the stability of a negative impedance converter are given. These conditions can be evaluated using standard computer-aideddesign software. Finally, a NIC prototype is given to validate and illustrate the presented design procedure, it is also integrated with a printed, blade-type, electrically small monopole in the VHF band.
\end{abstract}

\section{Keywords}

Negative Impedance Converter (NIC), stability, network Nyquist test, electrically small antennas

\section{Introduction}

Negative Impedance Converters (NIC) are electronic circuits that can provide new possibilities for radio-frequency and microwave circuits [1-4]. NICs can be used to increase the bandwidth of matching networks (MN), especially if they are realized on integrated or monolithic technologies (RFIC/MMIC). The NIC is a two-port network that, at port one, gives a negative, and possibly scaled, version of the impedance loading the port two; and vice versa.

The use of NICs can offer multiple possibilities to the circuit or system where they are integrated. Firstly, they can compensate the inductance or the capacitance to increase the impedance matching bandwidth of the circuit. Secondly, in parallel or series RLC circuits resonant circuits, negative capacitors or negative inductors can be used to modify the resonant frequency and the quality factor $Q$. Finally, negative resistors or negative conductances $G$ can be used to improve the $Q$ factor without modifying the resonant frequency.

One of the biggest drawbacks associated with the use of NIC circuits, at microwave frequencies, is their inherent stability problems. Several papers dealing with the circuit stability can be found [5-11]. All these methods are based on the Nyquist criterion to check if the corresponding network function does not have any poles lying in the righthalf of the complex plane (RHP).

The analysis of the stability of any microwave system requires both the study of a corresponding transfer function and the modeling of the inside active elements. Linear modeling of active circuits is preferred because of its speed and simplicity. Non-linear modeling requires both a heavier computational cost and accurate non-linear models of the active devices that are not always available [5-12]. For those reasons, when the corresponding network function is a suitable tool, and under some conditions [13], linear simulations can be used to research new design topologies using the $\mathrm{S}$ parameters of the active element.

Classic linear methods for NIC analysis can be classified in two groups: those related with the impedance, admittance or reflection coefficient function at the NIC output [6-10] and those related with the analysis of the open-loop gain in a two-port network [11], [14].

At microwave frequencies it is difficult to obtain the voltage or current transfer functions to apply to them the Nyquist criteria. For this reason, stability methods such as $K$-factor or $\mu$-factor, based on the $S$ parameters [15-18] have been used. However, although the simplicity of these methods, there is a lack of capability to linearly predict the NIC stability. Previously, in [19], the authors already stated that classic methods can provide inaccurate results when working with NICs, and that the safer way to test the stability of circuits involving non-foster elements (namely negative capacitors and negative inductors) is to assure that there are not any poles in the RHP. In addition, it was stated how stability also depends on the elements of the external passive circuit, and on the dispersive behavior of practical NICs implementations. However, the non-foster elements were approximated as ideal negative elements, while they are actually active circuits. In this paper a method that explores exhaustively the presence of poles in the RHP of any active system is presented. 
All the previous comments state that the stability analyses of circuits that include negative impedance converters are still completely open and more attention should be paid on them. In [20], [21] and [22], a new function for the stability analysis was proposed as a suitable tool to be used in the amplifier stability study. According to [21], the so-called normalized determinant function (NDF) allows using linear modeling for providing accurate methods to analyze the stability and performance of amplifier circuits. This paper proposes to use the NDF to analyze much more complex circuits such as negative impedance converters (NICs). In this method, the NIC is considered as a potentially stable device. Then, the goal is to find out which load and source impedances make the NIC stable. It must be emphasized that it is not only necessary to see which load impedance $Z_{\mathrm{L}}$ is negated, but also study how does the source impedance $Z_{\mathrm{S}}$ affect the NIC loaded with $Z_{\mathrm{L}}$. Other passive components in the system can also be included in the stability analysis, in this sense, a practical design example where the NIC is used as an embedded broadband matching network in an electrically small antenna (ESA), is presented in order to apply the NDF method for predicting the stability of the overall system: antenna $+\mathrm{MN}$.

The paper is organized as follows. Section 2 presents a critical study for the classic linear methods to analyze NICs in order to see its validity and shows the need of additional conditions for stability. In Sec. 3, a general method based on the normalized determinant function is presented. This method will allow us to study, in a general and an accurate way, the stability of the NIC circuits. Section 4 states the additional conditions that the methods presented in Section 2 must satisfy in order to be valid for a proper prediction of the stability. These additional conditions are based on the aforementioned NDF method. Then, to show the potential of the NDF, a practical design example is presented in Sec. 5. Finally, Section 6 gives the conclusions.

\section{Linear Methods for Studying Stabil- ity in NICs}

Two different kinds of methods are being used to analyze the NIC performance in this section: the reference plane methods and the loop gain method.

\subsection{Reference Plane Methods}

Concerning the reference plane methods, it was stated in [6] that the necessary condition for stability, at the input or output port when the other port was open or short-circuited, was that its corresponding impedance $Z(s)$ or admittance function $Y(s)$, respectively, had not any poles in the RHP, in other words, the real part of the one-port circuit function must be positive. There is a lack of consistency in the previous statement since there is not any comment on the actual loads to be inverted - capacitors and inductors; in addition, the active elements are considered as ideal ones leading to inaccurate results.

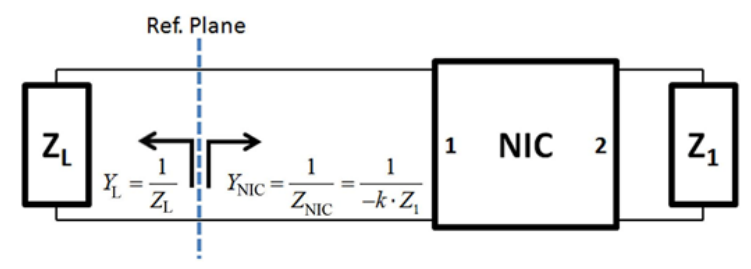

Fig. 1. Schematic of the NIC for a one-port network.

In a later approach [1], it was tried to generalize the previous criterion by considering any impedance or admittance to be inverted. A similar analysis was undertaken by considering the one-port network shown in Fig. 1. Then, the so called "open circuit stable" (OCS) condition states that the necessary, but not sufficient condition, for the NIC stability is that the absolute value of the impedance at port $1\left(Z_{\mathrm{L}}\right.$ in Fig. 1) should satisfy the following condition for all frequencies at which the NIC can effectively work.

$$
\left|Z_{\mathrm{L}}\right| \geq\left|Z_{\mathrm{IN}}\right|=\left|Z_{\mathrm{NIC}}\right| \text { or }\left|Y_{\mathrm{L}}\right| \leq\left|Y_{\mathrm{IN}}\right|=\left|Y_{\mathrm{NIC}}\right| \text { OCS port }
$$

While for the "short circuit stable" (SCS) condition, a necessary, but not sufficient condition for the NIC stability is that the absolute value of the admittance at port $1 Y_{\mathrm{L}}$ should satisfy the following condition for all frequencies at which the NIC can effectively work.

$$
\left|Y_{\mathrm{L}}\right| \geq\left|Y_{\mathrm{IN}}\right|=\left|Y_{\mathrm{NCC}}\right| \text { or }\left|Z_{\mathrm{L}}\right| \leq\left|Z_{\mathrm{IN}}\right|=\left|Z_{\mathrm{NIC}}\right| \text { SCS port . }
$$

For these reasons, the previous condition cannot assure the stability of the NIC device by itself, since it does not take into account the locus with frequency of the trace of the corresponding network function.

\subsection{Loop Gain Methods}

When the NIC is considered as a feedback system loaded with $Z_{\mathrm{L}}$ and $Z_{\mathrm{S}}$. The goal is to find out a set of values for $Z_{\mathrm{L}}$ and $Z_{\mathrm{S}}$ that makes the NIC stable. This method has been traditionally applied by the oscillator theory. If the NIC is considered as a feedback system, the lack of oscillating conditions makes the NIC stable. According to Fig. 2 the general network function is given as

$$
X_{\mathrm{o}}(s)=\frac{G(s)}{1+G(s) \cdot H(s)} \cdot X_{\mathrm{i}}(s) .
$$

where $X_{0}(s)$ and $X_{\mathrm{i}}(s)$ are the corresponding output and input functions (i.e. current or voltage) while $G(s)$ and $H(s)$ are the forward and reverse gain functions. The parallelseries feeding-back is used for NICs because one port is an impedance port while the other one is an admittance one.

The Nyquist stability criterion [23] describes the performance of the network by analyzing the placement of the zeroes and poles of the characteristic function $1+G(s) \cdot H(s)$. The zeroes of this function will be the of the closed loop function shown in (3). The necessary and sufficient condition for a stable system is that there are not any poles in the RHP. This condition has to be verified by looking at the Nyquist plot of the closed-loop gain functions. 


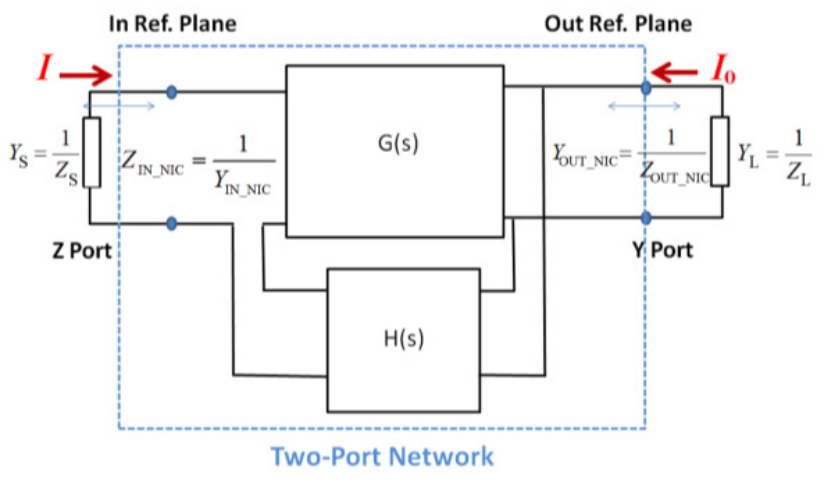

Fig. 2. Schematic of the NIC based on a feedback topology.

Another loop-gain method is presented in [24], where the closed-loop gain function was defined by the ratio between its input and output currents since any feedback system can be decomposed in a two-port network connected in closed loop (see Fig. 3). Then, the closed-loop transfer characteristic based on the $Z$ or $S$-parameters can be taken out as follows:

$$
\frac{I_{\mathrm{o}}}{I}=\frac{Z_{21}-Z_{11}}{Z_{11}-Z_{12}-Z_{21}+Z_{22}}=\frac{S_{22}-S_{11}+2 \cdot S_{21}-S_{12} \cdot S_{21}+S_{11} \cdot S_{22}-1}{1-\left(S_{12}+S_{21}-S_{12} \cdot S_{21}+S_{11} \cdot S_{22}\right)}
$$

where the $Z$ and $S$ parameters are the ones obtained from the two-port network being $I$ the input current and $I_{\mathrm{o}}$ the output current.

Assuming that the $S$-parameters do not present poles in the RHP, the stability of the NIC would be given by the existence of zeroes in the RHP of the denominator of the function $I_{\mathrm{o}} / I$.

Another alternative consists of using an open-loop gain function, instead of the close-loop gain function. The open-loop gain function can be obtained by opening the feedback loop at any point and applying the virtual ground technique [24]. Then, a new open-loop gain function $G_{\mathrm{L}}$ can be rewritten in terms of the $S$-parameters as in [24]:

$$
G_{\mathrm{L}}=\frac{Z_{21}-Z_{12}}{Z_{11}+Z_{22}-2 Z_{12}}=\frac{S_{21}-S_{12}}{1-S_{11} \cdot S_{22}+S_{12} \cdot S_{21}-2 \cdot S_{12}}
$$

The main advantages of this $G_{\mathrm{L}}$ function are its invariance with the opening point and that it does include the mismatching effect at the connection point. If $G_{\mathrm{L}}=1$, the root-loci of the $G_{\mathrm{L}}$ function define the existence of a pair of complex poles depending on how it encircles the +1 point. The system will be stable if the locus does not clockwise encircle the +1 point. It is important to remark that $G_{\mathrm{L}}=1$ is satisfied whenever:

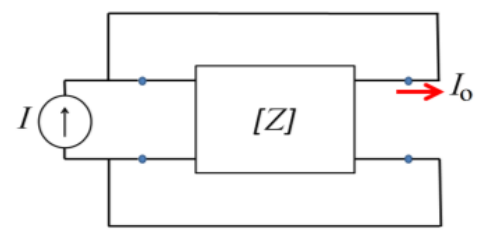

Fig. 3. Diagram of the NIC for calculating the currents ratio.

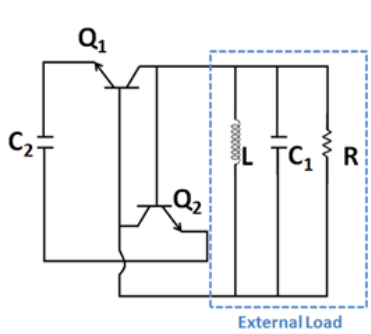

(a)

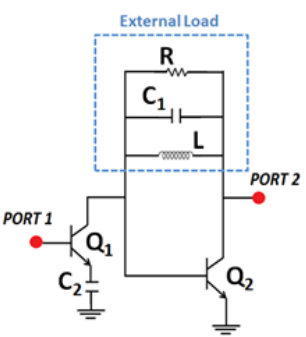

(b)
Fig. 4. (a) A classic NIC topology to convert $C_{2}$ where $C_{1}=5 \mathrm{pF}, R=495 \Omega, L=5 \mathrm{nH}, C_{2}=2 \mathrm{pF}, Q_{1}=Q_{2}=$ BFR360F, $V_{\mathrm{CE}}=3 \mathrm{~V}, I_{\mathrm{C}}=10 \mathrm{~mA}$. (b) The modified topology to analyze its stability with the closed loop gain function.

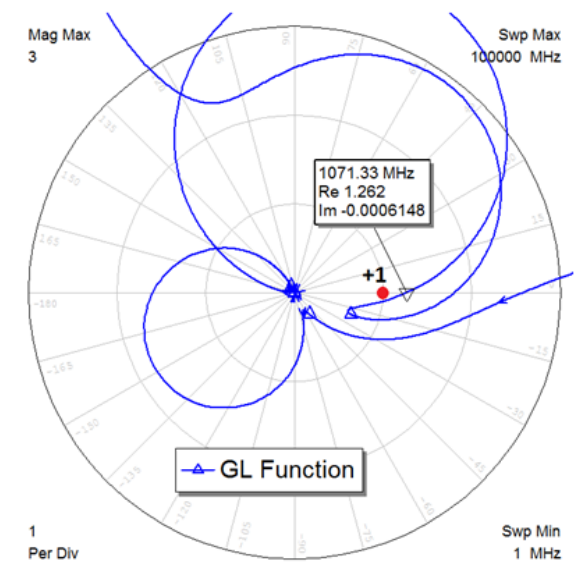

Fig. 5. Detail of the root-loci of the $\mathrm{G}_{\mathrm{L}}$ function.

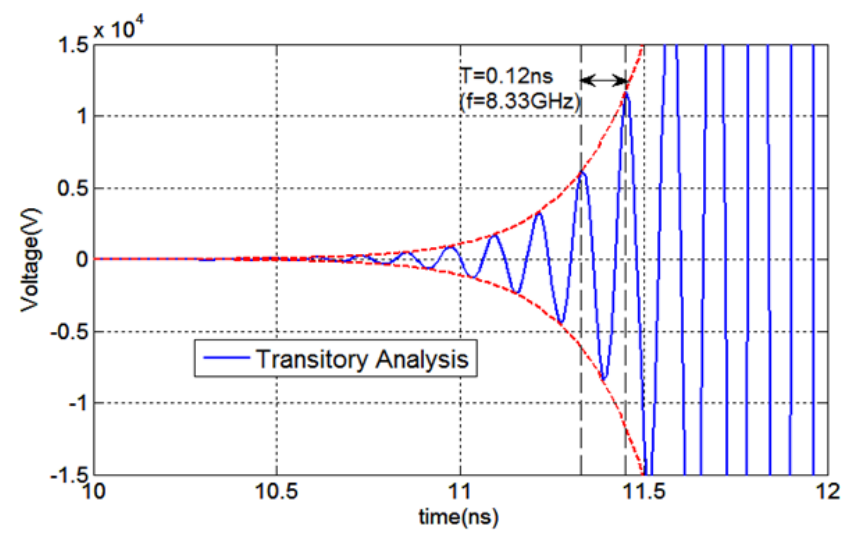

Fig. 6. Transient analysis response of the NIC structure depicted in Fig. 4(a)

$$
1-\left(S_{12}+S_{21}-S_{12} \cdot S_{21}+S_{11} \cdot S_{22}\right)=0
$$

which equals the denominator of the closed-loop transfer characteristic given in (4). Hence, $G_{\mathrm{L}}$ describes the poles of the closed-loop network. In order to demonstrate the validity of the previous analysis, one of the classic NICs shown in [2] to invert the capacitance $C_{2}$ in Fig. 4(a), is analyzed. This scheme shows the external load connected to the NIC which is a parallel connection of a capacitor, an inductor and a resistance $(L, C 1, R)$. When the closed loop is opened, at the point connecting the collector and base of one of the bipolar transistors, it yields to the circuit shown in Fig. 4(b). 
Figure 5 shows a detailed polar plot of the open loop $G_{\mathrm{L}}$ function as frequency varies. It can be seen that the $G_{\mathrm{L}}$ passes through the real axis at $x=1.262$ corresponding to $1.071 \mathrm{GHz}$. It can be seen that the $G_{\mathrm{L}}$ does not encircle the +1 point. According to the proposed criterion given through (5), the proposed NIC is stable, agreeing with the loop gain method for the considered load conditions.

In addition to the performed frequency analysis, a time analysis is performed, by means of the $\mathrm{AWR}^{\circledR}$ software, to see the behavior of the NIC with a transient signal (see Fig. 6). These results show that the circuit is clearly unstable and oscillates at $8.33 \mathrm{GHz}$. In spite of the fact that loop gain method predicted a stable performance, the transient analysis predicts an unstable response. Then, it seems that additional conditions must be added to the previous analysis in order to correctly predict the NIC stability.

\section{Normalized Determinant Function (NDF)}

From the previous analysis, it can be concluded that additional conditions are required for a correct prediction of the NIC stability. In this sense, the so-called normalized determinant function (NDF) ([20] and [21]) can be considered as the only single step method able to predict in a rigorous way the stability of any N-port network structure. The NDF was proposed as a method to verify the Rollet proviso conditions in two-port amplifier networks [25].

The goal is, as before, to find out the set of values for $Z_{\mathrm{L}}$ and $Z_{\mathrm{S}}$ that make the NIC stable. The NDF is defined as the ratio between the determinant of the network function when all the dependent generators are switched on $\Delta(s)$ and the determinant of the network function when all the generators are switched off $\Delta_{0}(s)$ :

$$
N D F=\frac{\Delta(s)}{\Delta_{0}(s)} .
$$

The NDF has some important properties: as the active network behaves as the passive network when $\omega$ or $\sigma$ tends to infinity, the NDF tends to one. The NDF has not any poles in the RHP since $\Delta_{0}(s)$ is a passive network and the denominator of $\Delta(s)$ and $\Delta_{0}(s)$ are equal. Taking these properties into account, and using the principle of the argument theorem, it is possible to determine the number of zeroes of the NDF in the RHP (poles of the system in the RHP), as equal to the number of clockwise encirclements, around the origin, that the NDF makes, when it is evaluated from $\omega=-\infty$ to $\omega=\infty$. Therefore, the system will be stable if, and only if the NDF does not clockwise encircle the origin of the complex plane.

One of the advantages of the NDF is that it can be easily calculated through the return ratio functions given by Bode. Then the NDF can be given as follows:

$$
N D F=\prod_{i=0}^{k}\left(R R_{i}+1\right)
$$

where $R R_{i}$ is the return ratio for the $i^{\text {th }}$ dependent source; $k$ is the total number of active elements (dependent sources) of the network; $R R_{1}$ is the return ratio of the source $i=1$, when the sources $i=2,3, \ldots, k$ are switched on; $R R_{2}$ is the return ratio of the source $i=2$, when the source $i=1$ is switched off, and sources $i=3,4, \ldots, k$ are switched on; and $R R_{3}$ is the return ratio of the source $i=3$, when the sources $i=1,2$, are off, and sources $i=4,5, \ldots, k$ are on. In the same way, $R R_{k}$ is the return ratio of the source $i=k$, when the sources $i=1,2, \ldots, k-1$ are switched off. Finally, to calculate each $R R_{i}$, the dependent source in the trans-conductance model, of the $i^{\text {th }}$ active element, must be replaced with the expression $-g_{\mathrm{m}} V_{i}$ as it is shown in Fig. 7. Then, evaluating the response of its dependent node to an external excitation $V^{\prime}$ of magnitude 1 , the $R R_{i}$ can be calculated as:

$$
R R_{i}=-\frac{V_{i}}{V^{\prime}}
$$

In order to use the NDF, or the $R R$, it is necessary to have a linear model of the transistor or to extract it from the non-linear model or the $S$-parameters of the device. Figure 7 shows the equivalent circuit of a bipolar transistor without any parasitic elements, all the parasitic elements of the transistor are included in the feed-back network. This circuit allows the access to any internal port, so that the RR can be calculated with the circuit simulator. The NDF can be directly calculated with any circuit simulator and it is able to detect the number of poles of the network function. There must not exist any poles in the RHP for a stable NIC.

The main drawback of the NDF is that it requires a prior full linear modeling of each active device around its biasing point, which can be difficult and inaccurate at high frequencies. Other approaches are possible using nonlinear models, but extracting the small signal model and using the NDF is generally the safest one [25].

Now, an analysis example is presented to show how the NDF can predict the potential instabilities of NIC circuits. The circuit previously described in Sec. 2.2 will be studied. Figure 8 shows the NDF of the circuit, where it can

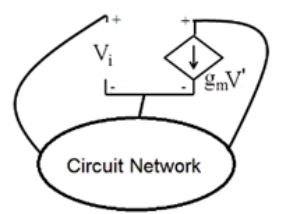

Fig. 7. Linear equivalent circuit for the bipolar transistor.

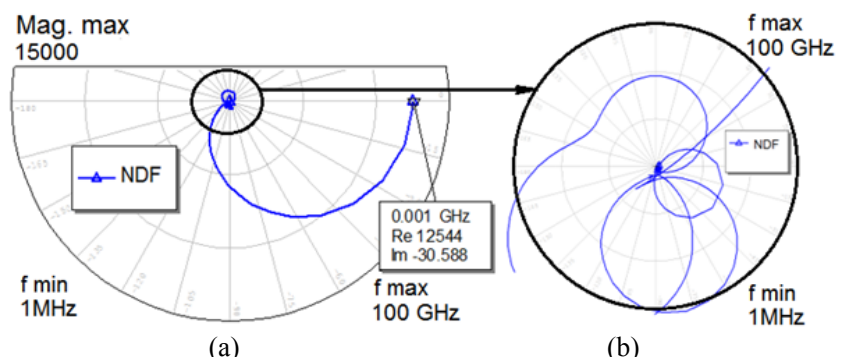

Fig. 8. a) NDF of the NIC circuit proposed in Sec. 2.2. b) Zoom around the origin. 
be clearly observed how the NDF encircles the origin in a clockwise sense, so the circuit is unstable as it was previously stated. In addition, the predicted oscillation frequency is around $7.45 \mathrm{GHz}$ (the NDF crosses through the negative real axis at that frequency), very close to the one predicted by the transient analysis performed and depicted in Fig. 6. This small discrepancy comes from non-linear effects that modify the original linear $R R_{i}$ functions and, subsequently, the NDF accuracy.

\section{Unified Approach between NDF and Classic Methods for Stability Analysis of Non-Foster Forms}

It is very useful to work with the impedances or reflection coefficient during the NICs design. However, it must be pointed out that additional conditions (the proviso) must be defined and satisfied before using any of the methods proposed in Sec. 2. In other words, the classic methods can only properly check the stability if the proviso is satisfied [25-27]. The next subsection shows which are these additional conditions for the methods proposed in Sec. 2, and how they can easily be evaluated using the NDF.

\subsection{Additional Conditions for the Reference Plane Methods}

In this section the additional conditions that must be satisfied in order that the reference plane methods studied in Sec. 2.1 are derived to properly predict the stability. Once these additional conditions are fulfilled, the Nyquist analysis of the characteristic equations $(Z, Y)$ can predict the presence of poles in the RHP, and hence the stability of the loaded network.

Firstly, the admittance criterion is used, studying the function $Y_{\mathrm{T}}(\omega)$, where $Y_{\mathrm{T}}(\omega)=Y_{\mathrm{NIC}}(\omega)+Y_{\mathrm{L}}(\omega)$, being $Y_{\mathrm{NIC}}$ the admittance provided by the NIC and $Y_{\mathrm{L}}$ the load connected to the NIC) (see Fig. 1). The analysis in the frequency domain of $Y_{\mathrm{T}}(\omega)$ is performed to obtain the Nyquist plot of $Y_{\mathrm{T}}(\omega)$. The Nyquist criterion provides the number $N Z-N P$, where $N Z$ and $N P$ are respectively the number of zeros and poles in the RHP. This number $(N Z-N P)$ corresponds with the number of times that the function $Y_{\mathrm{T}}(\omega)$ encircles the origin of the complex plane when it is evaluated from $\omega=-\infty$ to $\omega=\infty$.

To be sure that the Nyquist analysis of $Y_{\mathrm{T}}(\omega)$ provides the number of poles of the system, it is needed that $Y_{\mathrm{T}}(\omega)$ has not any poles in the RHP. The necessary condition is that $Y_{\mathrm{NIC}}$ has not any poles in the RHP (visible or hidden ones). It is very important to point out that the function $Y_{\mathrm{NIC}}$ is a reduced function of a bigger network, hence it might not present hidden poles (poles of the system that are not present in $Y_{\mathrm{NIC}}$ since it is a reduced function), that can invalidate the Nyquist analysis of $Y_{\mathrm{T}}$. On the other hand, $Y_{\mathrm{L}}$ will not present any pole in the RHP, because it is a passive network.
The proviso to check before using the admittance criterion is that the $Y_{\mathrm{NIC}}$ network loaded with short-circuit has not any poles in the RHP. It is that the NDF does not clockwise encircle the origin ([8] and [25]) (see Fig. 1) as it was explained in Sec. 3 .

The additional required conditions when working with the impedance criteria (function $Z_{\mathrm{T}}(\omega)$ ) can be derived in a similar way: $Z_{\mathrm{NIC}}$ must not present any poles in the RHP when the network is loaded with open-circuit. Once this additionally condition is verified by using the NDF, the Nyquist criteria can be used over $Z_{\mathrm{T}}$ to study the stability of the circuit.

Finally, if the reflection coefficient is used, the additional condition is that $\Gamma_{\mathrm{NIC}}$ has not any poles in the RHP when the network is loaded with $Z_{0}$. As in the previous cases, it can be easily done using the NDF.

\subsection{Additional Conditions for the Loop-Gain Method}

In this section the additional conditions for the openloop methods are derived. These methods include the stability factor $K$ and the methods which use the open-loop gain (see Sec. 2.2).

Firstly, the stability factor methods are used. The additional condition for the stability factor methods is that the network has not any poles in the RHP when it is loaded with $Z_{0}$. This analysis can be performed using the NDF when the circuit is loaded with $Z_{0}$.

To illustrate this point, the circuit shown in Fig. 9 [4] is studied. The circuit consists on a floating NIC connected between a small antenna and the port $Z_{0}$ (typically $50 \Omega$ ). In this case the NIC is used to negate the impedance $1 /(j \omega C)+j \omega L_{1}$ in order to compensate the reactance of the small antenna and hence improve the bandwidth. However, Figure 10 shows that the circuit does not satisfy the suitable proviso, since the NDF encircles the origin when the circuit is loaded with $Z_{0}$, so the classic stability factor (i.e. Rollet factor or $\mu$-factor) cannot be used to predict the stability.

The second method under study is the one based on the open-loop gain. This method states that the circuit is stable if the denominator of (3) has not any zeroes in the RHP, or, what it is the same, that the function $G(\omega) \cdot H(\omega)$ does not encircle the -1 point (Nyquist theorem).

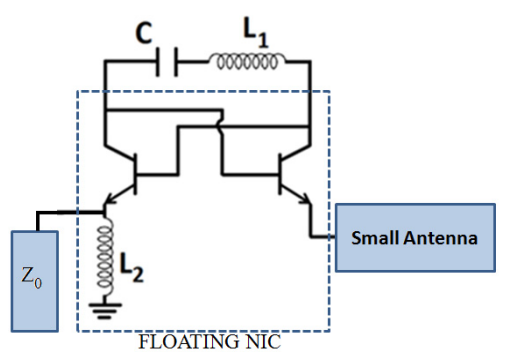

Fig. 9. A basic NIC topology connected to a small antenna, where: $\mathrm{C}=7.5 \mathrm{pF}, \mathrm{L}_{1}=291.92 \mathrm{nH}$ and $\mathrm{L}_{2}=55 \mathrm{nH}$. 


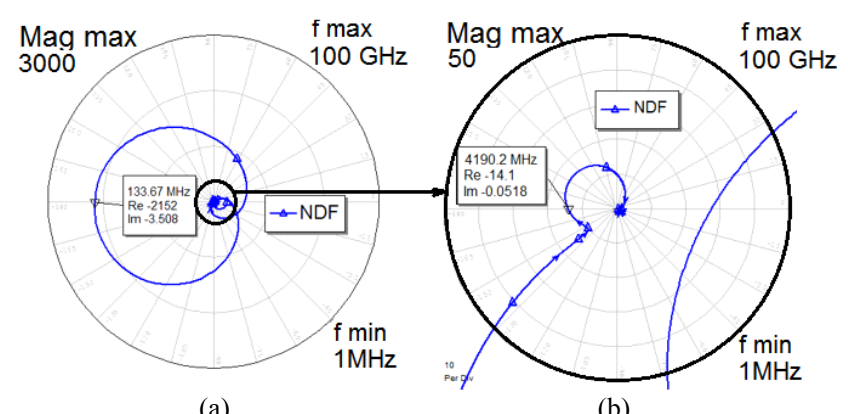

(a)

(b)

Fig. 10. a) NDF of the circuit under study.

b) Zoom around the origin.

However, before using the $G_{\mathrm{L}}$ function (5) to properly predict the existence, or not, of poles in the RHP, some additional conditions must be verified:

- The first condition states that $G_{\mathrm{L}}$ must not have any poles in the RHP. It does not have any poles if the test function $T F=1-S_{11} \cdot S_{22}+S_{12} \cdot S_{21}-2 \cdot S_{12}$ has not any zeroes in the RHP. This can be done by performing the Nyquist analysis of the $T F$ and checking that it does not encircle the origin in a clockwise sense.

- The second one is that the numerator of $I_{\mathrm{o}} / I$ (4) must not have any poles (visible or hidden) in the RHP. As in the previous case, it will not have any poles if none of the $S$ parameters have any poles in the RHP and, in addition, the TF has not any zeroes in the RHP (first condition).

In practice, these two aforementioned conditions can be tested as follows. Firstly, the NDF must be calculated over the open-loop network loaded with $Z_{0}$ to test that $S$ parameters have not any poles in the RHP. Secondly, the Nyquist analysis over the $T F$ (denominator of $G_{\mathrm{L}}$ ) must be undertaken. If both tests are positive, none of them encircle the origin, then $G_{\mathrm{L}}$ can be used to predict the existence or inexistence of poles of the function $I_{\mathrm{o}} / I$.

As an example, it is shown how the circuit presented in Sec. 2.2 (see Fig. 4(a), (b)) does not satisfy the proviso, so the $G_{\mathrm{L}}$ function cannot be used to predict the stability. Figure 11 (a), (b) shows how the first part of the aforementioned proviso conditions is not satisfied (NDF when the network is loaded with $Z_{0}$ ) because the NDF does encircle the origin. Moreover, the second part of the proviso is not fulfilled because the Nyquist analysis of the $T F$ indicates

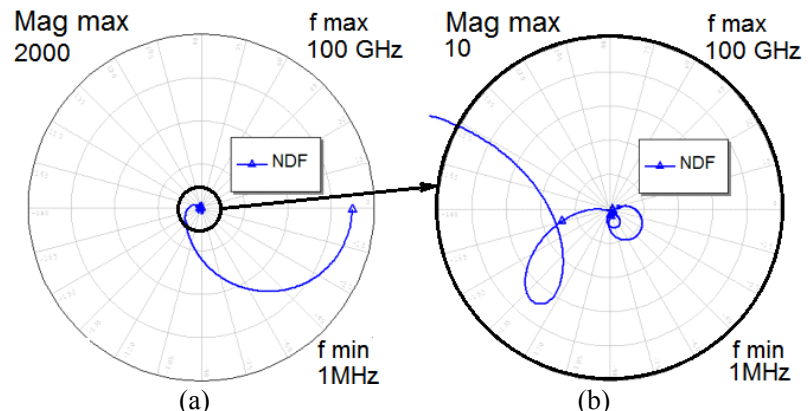

Fig. 11. a) NDF of the circuit proposed in Sec. 2.2. b) Zoom around the origin.

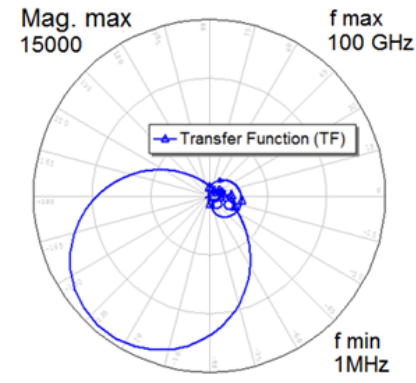

Fig. 12. Nyquist analysis of the transfer function TF.

the existence of zeros (poles of the system) in the RHP, as it can be observed in Fig. 12. For this reason, as the proviso is not satisfied, the open-loop gain $G_{\mathrm{L}}$ [24] cannot be used as a predictor of the stability, and it provides incorrect results as it was shown in Sec. 2.2 using the transient analysis (see Fig. 6).

\section{Design Example}

In this section it will be shown how the NDF can be used to predict the stability of a NIC during the linear design step. The NIC is designed to present a negative capacitor and the chosen topology is the one presented in Fig. 13. It is based on a silicon N-channel dual-gate MOSFET transistor (BF998) with a transconductance $g_{\mathrm{m}}$ of $25 \mathrm{mS}$ at the selected bias point $\left(Q_{2}\right.$ and $Q_{3}$ in Fig. 13(a) connected in a ring topology, and a load $Z_{\mathrm{L}}$ to be inverted). Additionally, two more transistors $\left(Q_{1}\right.$ and $Q_{4}$ in Fig. 13(a)) are used as currents sources for biasing purpose.

Using a small-signal analysis, the equivalent circuit of the NIC can easily be obtained as it can be seen in Fig. 13(b). Moreover, the effect of $R_{\mathrm{DS}}$ and $C_{\mathrm{GS}}$ can be neglected for low frequencies, in such a way that the input impedance can be approximated as (10), where $R_{\mathrm{n}}=-1 /\left(g_{\mathrm{m}}^{2} \cdot R_{\mathrm{DS}}\right)$ and $L_{\mathrm{n}}=-C_{\mathrm{GS}} / g_{\mathrm{m}}^{2}$ are parasitic negative resistance and negative inductance respectively (being $g_{\mathrm{m}}$ the FET transconductance, $C_{\mathrm{GS}}$ the gate-source capacitance and $R_{\mathrm{DS}}$ the drain-source resistor of the FET small signal model).

$$
Z_{\mathrm{IN}}=R_{\mathrm{n}}+j \omega L_{\mathrm{n}}+\frac{-1}{Z_{\mathrm{L}} \cdot g_{\mathrm{m}}^{2}}
$$

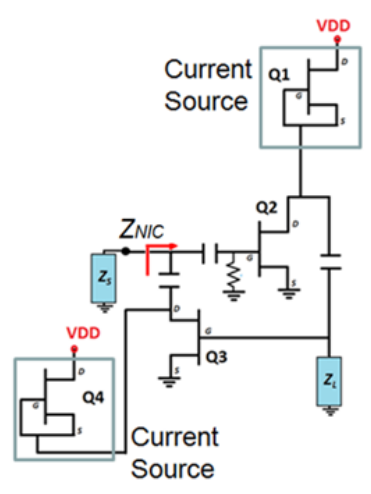

(a)
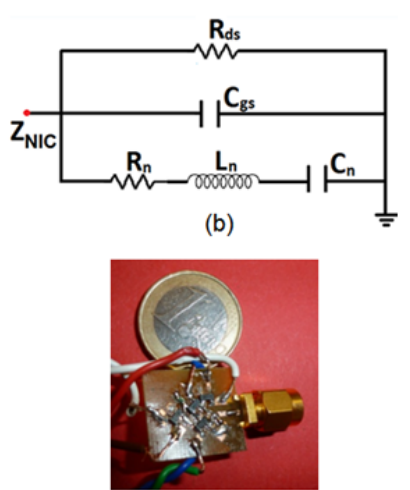

(c)
Fig. 13. a) Antenna sketch with the embedded NIC. b) NIC topology and its c) equivalent circuit. 
As an isolated negative capacitor is always unstable, an additional load $Z_{\mathrm{S}}$ is required to make the system stable. In practice, this load $Z_{\mathrm{S}}$ is the one connected to the NIC inside the application. For instance, if a NIC is used to improve the matching of an electrically small antenna (ESA) the load $Z_{\mathrm{S}}$ will be the impedance of the antenna at the port where it will be connected. The NDF can be used during the design to determine which loads, $Z_{\mathrm{S}}$, make the circuit stable as it will be shown below. Figure 13(c) shows the prototyped circuit.

The simulations are performed using a real (not lossless) inductor model of $56 \mathrm{nH}$ (Coilcraft ${ }^{\circledR} 0805 \mathrm{CS}$ $L=56 \mathrm{nH}, \quad R_{1}=13 \Omega, \quad R_{2}=0.34 \Omega, \quad C=0.094 \mathrm{pF}$, $K=0.000101 \sqrt{ } f$ ) and a quality factor of 60 . As the inductance has a value of $56 \mathrm{nH}$ the expected negative capacitance is about $C_{\mathrm{n}}=-32 \mathrm{pF}$, according to (8). Taking this into account, an analysis of the circuit is performed using the NDF. This analysis shows that the circuit is stable when $Z_{\mathrm{S}}$ is a positive capacitor larger, in magnitude, than the negative one $(-32 \mathrm{pF})$ and it is unstable in any other case:

$$
\begin{aligned}
& C_{\mathrm{s}}>\left|C_{\mathrm{n}}\right| \rightarrow \text { Stable, } \\
& C_{\mathrm{s}} \leq\left|C_{\mathrm{n}}\right| \rightarrow \text { Unstable. }
\end{aligned}
$$

To illustrate this behavior, Figure 14 shows the NDF for two values of the $C_{\mathrm{s}}$ capacitor. For $C_{\mathrm{s}}=50 \mathrm{pF}$ the NDF does not clockwise encircle the origin so the circuit is stable. But in the case of $C_{\mathrm{s}}=25 \mathrm{~F}$ the NDF clearly encircles the origin, so the circuit is unstable. Similar stability criteria have been obtained in [28], but the authors consider that the NDF is a more general tool, valid not only for the topology of this example but for any other topology. In addition, the use of the NDF also takes into account many effects such as the parasitic effects, the effect of the transmission lines, the biasing networks, the non-idealities of the components, etc. that were not considered in [28].

This behavior was also experimented in practice with the manufacturing of a prototype. It can be observed in the measurements obtained with the spectrum analyzer that the behaviors of the manufactured circuits are the expected ones Figure 15(a) corresponds to the stable case of $\left(C_{\mathrm{S}}=50 \mathrm{pF}>\left|C_{\mathrm{n}}\right|\right)$. In this case the circuit is stable since there is not any signal apart from small peaks corresponding to DCS \& UMTS and radio interferences. On the other hand, Figure 15(b) shows the spectrum of the unstable case

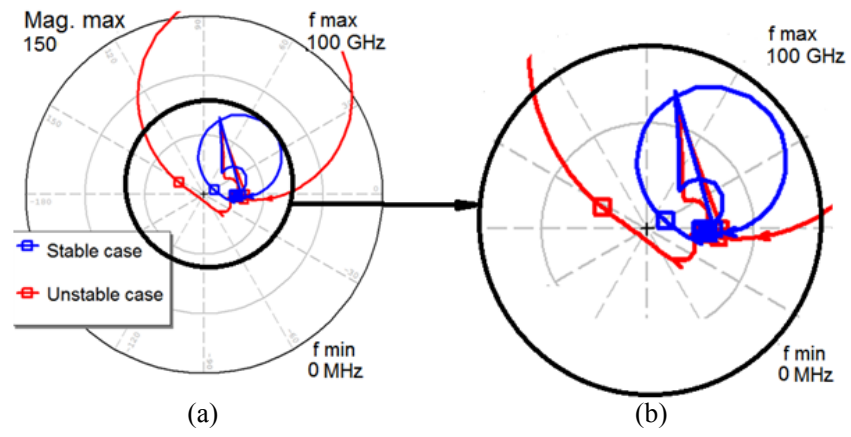

Fig. 14. a) NDF of the proposed design example. b) Zoom around the origin.

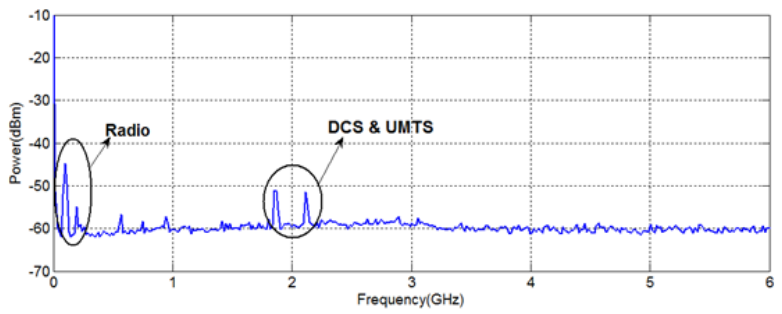

(a)

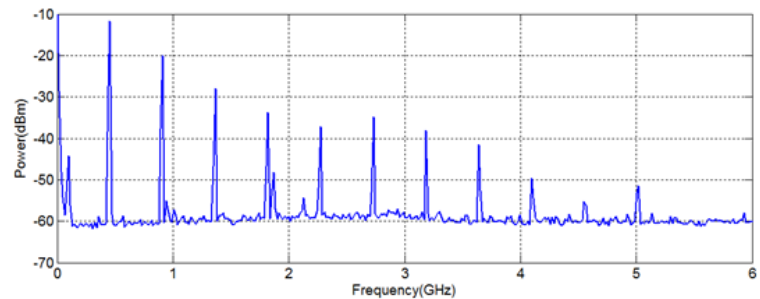

(b)

Fig. 15. Spectrum. a) Stable case $\left(C_{\mathrm{S}}=50 \mathrm{pF}\right)$. b) Unstable case $\left(C_{\mathrm{S}}=25 \mathrm{pF}\right)$.

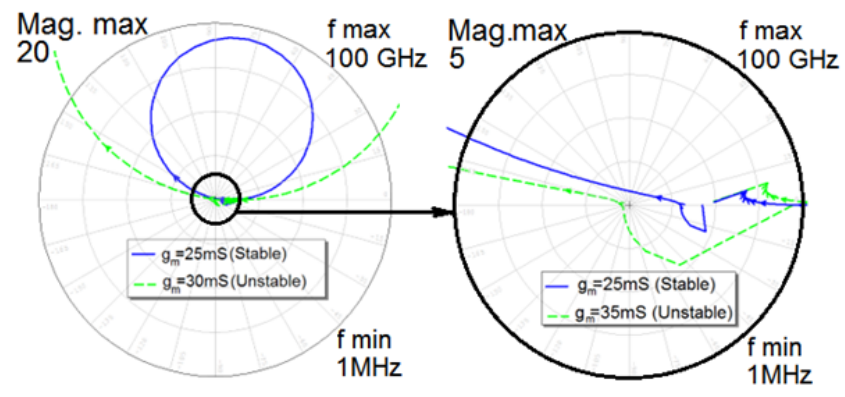

(a)

(b)

Fig. 16. a) NDF of the circuit. b) Zoom around the origin.

$\left(C_{\mathrm{S}}=25 \mathrm{pF}<\left|C_{\mathrm{n}}\right|\right)$. In this case the circuit is clearly unstable and oscillates at $455 \mathrm{MHz}$ with importance levels in its harmonics.

In addition, the prototype is unstable for $C_{\mathrm{S}}=100 \mathrm{pF}$ and high biasing voltages, which was supposed to be stable. The NDF can also predict this behavior: When the biasing voltage increases, the drain-source voltages of the FETs also do, and therefore the transconductance of the FET can also increase slightly.

Figure 16 shows the NDF of the circuit for two values of the transconductance and it can be observed how when the transconductance increases from $25 \mathrm{mS}$ to $30 \mathrm{mS}$ the circuit turns unstable (the NDF clockwise encircles the origin) as it was observed in the prototype.

Once the circuit is stable, it can be measured with the network analyzer in order to obtain the input impedance of the NIC, and test if it is really working as a negative capacitor. The measurements of the NIC, after de-embedding the positive capacitor and the effect of the connector, is shown in Fig. 17. It can be observed how the circuit behaves as a negative capacitor of $(-34 \pm 4) \mathrm{pF}$, very close to the $-32 \mathrm{pF}$ theoretically predicted up to $200 \mathrm{MHz}$. For higher frequencies the NIC does not work properly and it turns to a conventional positive capacitor as it was expected. The differences between measurements and simu- 


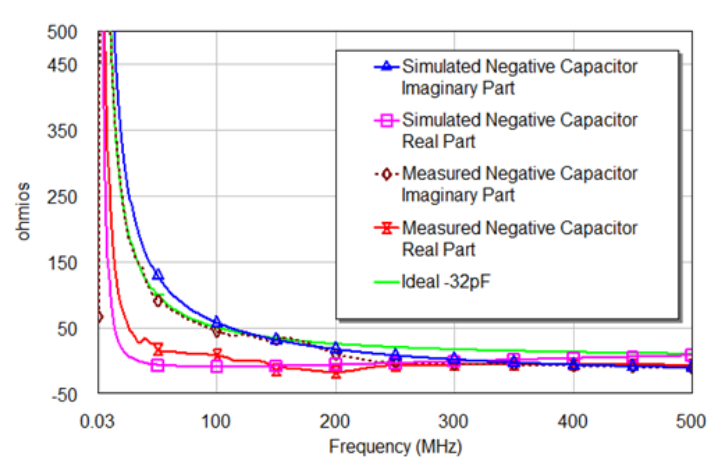

(a)

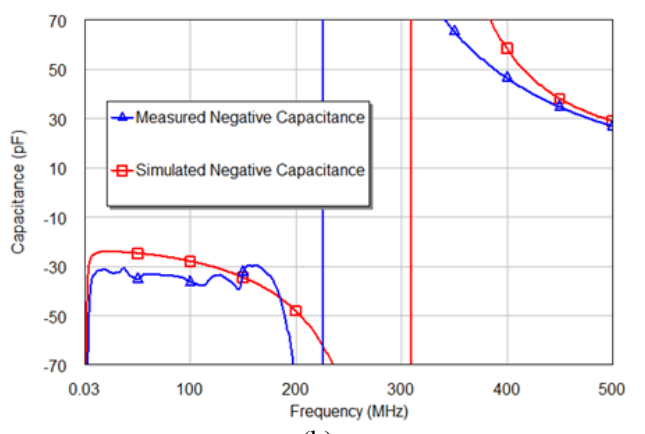

(b)

Fig. 17. NIC measurements. a) NIC impedance. b) Equivalent capacitance.

lations are due to the fact that to obtain a negative capacitor depends on the FET transconductance, which is difficult to characterize in practice.

The prototype presented here works up to $200 \mathrm{MHz}$ due to the fact that as a rule of thumb NICs only work up to a frequency ten times smaller than the transient frequency of the transistors [30]. The transistor employed here (BF998) has a transient frequency of $1.9 \mathrm{GHz}$, so the prototype presents the expected bandwidth. A FET transistor has been chosen instead of other with a higher transient frequency because it is easier to obtain accurate linear models at low frequencies to show the validity of the method. At high frequencies, more feedback paths, and more parasitic effects must be considered, so obtaining linear models is more complicated.

However, it is important to remark that although the method has only been validated with a NIC working up to $200 \mathrm{MHz}$, the method is general and valid no matter the operation frequency as long as accurate linear models of the active devices, passive elements, interconnections are available.

For instance, this NDF method can be used for MMIC where interconnections are not too lossy. Of course this method can also be used for or in Silicon and SiGe processes where the interconnections are lossy and frequency dependent, including the influence of the layout in the linear model.

For the sake of completeness, the designed and prototyped NIC circuit is used as an embedded active matching network, for a blade-type small monopole of $22 \mathrm{~cm} \times 25 \mathrm{~cm}$ over a FR4 substrate of $1.6 \mathrm{~mm}$ in width and $\varepsilon_{\mathrm{r}}=4.3$. The intended impedance matching bandwidth

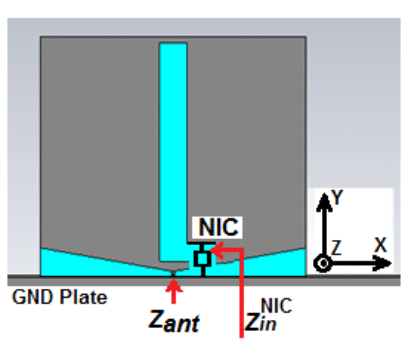

(a)

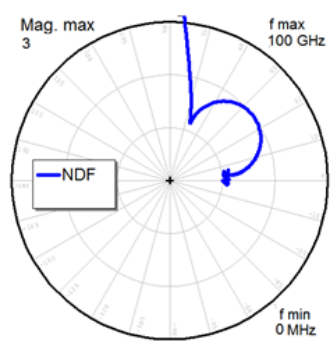

(b)
Fig. 18. Antenna sketch with the embedded NIC. b) NDF of the whole design: antenna+NIC.

(i.e. $\left.\left|S_{11}\right|<-8 \mathrm{~dB}\right)$ is over a $2: 1$ range $(50 \div 110 \mathrm{MHz})$. Figure 18(a) shows the antenna structure with the embedded MN, a vertical slot has been included to force a longer way for the current along the monopole with the purpose of reducing the electrical size of the antenna at the intended frequency range. The same NIC topology, as the one presented in the previous example, is selected for acting as an active $\mathrm{MN}$, but this time with a reactance expected to realize an ideal negative inductor, in order to compensate the capacitive nature of the monopole impedance at lower frequencies. The whole antenna system (antenna+NIC) stability is evaluated by means of the NDF (see Fig. 18(b)), predicting stability.

$Z_{\text {in }}^{\text {NIC }}$ has to be chosen according to the impedance needed at the connecting point in the monopole structure, in order to obtain an input impedance $\left(Z_{\text {in }}{ }^{\text {ant }}\right)$ as near as possible to the system impedance $(50 \Omega)$. The active matching antenna method, proposed in [29], can be applied in this case, to calculate an analytical impedance $\left(Z_{\mathrm{MN}}\right)$ to

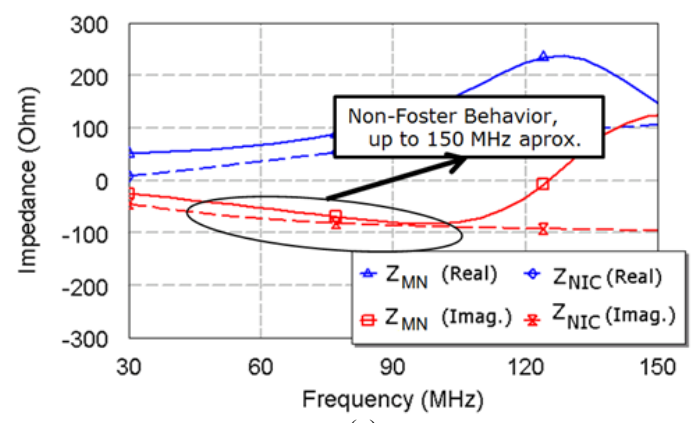

(a)

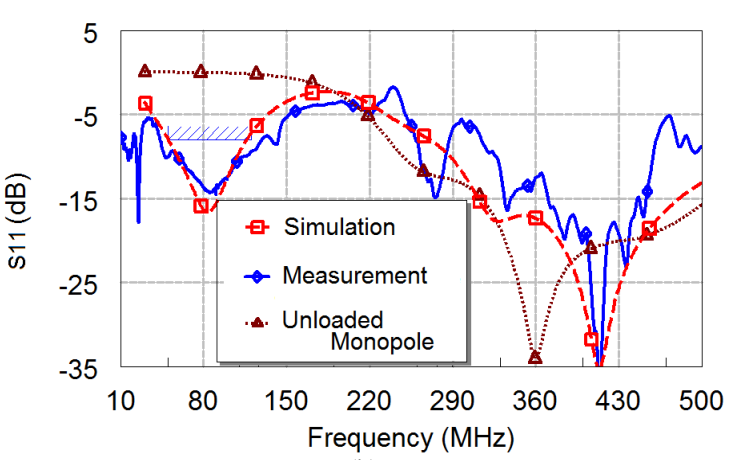

(b)

Fig. 19. a) Computed $Z_{\mathrm{MN}}$ response and the simulated $Z_{\mathrm{NIC}}$. b) The $S_{11}$ parameter at the input port for both cases: the unloaded and the active loaded monopole with the MOSFET-based NIC. 
be realized with our NIC circuit. Once the analytical impedance $Z_{\mathrm{MN}}$ is computed, it is possible to choose a capacitor to load the NIC $\left(Z_{\mathrm{NIC}}=1 / j \omega C\right)$, then a negative inductor, proportional to the value of the capacitance $C$, is obtained $L_{\mathrm{n}}=-g_{\mathrm{m}}{ }^{2} \cdot C$. Figure 19(a) shows the computed analytical $Z_{\mathrm{NIC}}$ and the simulated NIC input impedance $Z_{\mathrm{IN}}$.

The new engineered impedance matching band is obtained at VHF frequencies, as it is shown in Fig. 19(b) where a fractional impedance bandwidth $(F B W)$ of $63 \%$ is obtained. One more advantage of the NDF method stands in the fact that it is possible to include the antenna $S$-parameter model in the stability analysis.

\section{Conclusion}

This paper proposes the use of the Normalized Determinant Function (NDF) as a tool to analyze the stability of NIC circuits (or any circuit in general). The NDF can easily take into account different effects such as the parasitic effects, the non-idealities, the effect of the biasing networks, the effect of the transmission lines, etc. It has been shown that classic stability methods such as reference plane, stability factor $(K$ or $\mu)$ or open-loop gain methods can yield to incorrect results. All the classic methods work with parameters extracted from reduced networks, so some poles of the complete networks could be missed and then they cannot be detected. For these reasons, these methods cannot be considered by themselves as universal methods for the stability analysis. However, if some additional conditions were satisfied, the so called proviso conditions, all these methods can still work properly. All these necessary additional conditions are based in the NDF. Nevertheless, the authors conclude that the analysis based on the NDF always yields correct predictions regarding the stability and the oscillation frequency and, hence, it is the safer and quicker option for the designers. On the other hand, the main drawback of the NDF for the NIC design is that the accurate linear models of the active devices are required.

Finally, the proposed NDF method has been used to successfully design a negative capacitor working up to $200 \mathrm{MHz}$. It has been shown how the NDF predicts the loads and the biasing conditions that make the circuits stable or unstable and how these predictions are in perfect agreement with the performed measurements.

\section{References}

[1] SUSSMAN-FORT, S.E., RUDISH, R.M. Non-foster impedance matching of electrically-small antennas. IEEE Transactions on Antennas and Propagation. 2009, vol. 57, no. 8, p. 2230-2241. ISSN 0018-926X, 1558-2221. DOI:10.1109/TAP.2009.2024494

[2] SUSSMAN-FORT, S.E., RUDISH, R.M. Non-foster impedance matching for transmit applications. In IEEE International Workshop on Antenna Technology Small Antennas and Novel Metamaterials. New York (USA), March 2006, p. 53-56. DOI: 10.1109/IWAT.2006.1608973

[3] SUSSMAN-FORT, S.E. Non-Foster vs. active matching of an electrically-small receive antenna. In 2010 IEEE Antennas and
Propagation Society International Symposium. Toronto (ON, Canada), 2010, p. 1-4. DOI: 10.1109/APS.2010.5562052

[4] ABERLE, J.T. Two-port representation of an antenna with application to non-foster matching networks. IEEE Transactions on Antennas and Propagation. 2008, vol. 56, no. 5, p. 1218-1222. ISSN 0018-926X. DOI: 10.1109/TAP.2008.922173

[5] SUÁREZ, A., QUÉRÉ, R. Stability Analysis of Nonlinear Microwave Circuits. Artech House, 2003. ISBN 978-1-58053-586-1.

[6] BROWNLIE, J.D. On the stability properties of a negative impedance converter. IEEE Transactions on Circuit Theory. 1966 , vol. 13, no. 1, p. 98-99. ISSN 0018-9324. DOI: 10.1109/TCT.1966.1082542

[7] HOSKINS, R. F. Stability of negative-impedance convertors. Electronics Letters. 1966, vol. 2, no. 9, p. 341-341. ISSN 1350911X. DOI: 10.1049/el:19660287

[8] BROWNLIE, J. D. Small-signal responses realizable from d.c.biased devices. Proceedings of the Institution of Electrical Engineers. 1963, vol. 110, no. 5, p. 823-829. ISSN 0020-3270. DOI: $10.1049 /$ piee. 1963.0111

[9] STERN, A. P. Stability and power gain of tuned transistor amplifiers. Proceedings of the IRE, 1957, vol. 45, no. 3, p. 335-343. ISSN 0096-8390. DOI: 10.1109/JRPROC.1957.278369

[10] DAVIES, A. Stability properties of a negative immittance converter. IEEE Transactions on Circuit Theory, 1968, vol. 15, no. 1, p. 80-81. ISSN 0018-9324. DOI: 10.1109/TCT.1968.1082775

[11] MIDDLEBROOK, R.D. Measurement of loop gain in feedback systems. International Journal of Electronics Theoretical and Experimental. 1975, vol. 38, no. 4, p. 485-512. ISSN 0020-7217. DOI: $10.1080 / 00207217508920421$

[12] VENDELIN, G., PAVIO, A.M., ROHDE, U.L. Microwave Circuit Design using Linear and Nonlinear Techniques. $2^{\text {nd }}$ ed. New York: John Wiley \& Sons, 1990 [accessed 24th May, 2016]. ISBN 9780-471-41479-7.

[13] JACKSON, R.W. Rollett proviso in the stability of linear microwave circuits-a tutorial. IEEE Transactions on Microwave Theory and Techniques, 2006, vol. 54, no. 3, p. 993-1000. ISSN 0018-9480. DOI: 10.1109/TMTT.2006.869719

[14] BODE, H. W. Network Analysis, Feedback Amplifier Design. New York, USA: Van Nostrand, 1945.

[15] ROLLETT, J. Stability and power-gain invariants of linear twoports. IRE Transactions on Circuit Theory, 1962, vol. 9, no. 1, p. 29-32. ISSN 0096-2007. DOI: 10.1109/TCT.1962.1086854

[16] EDWARDS, M. L., SINSKY, J.H. A new criterion for linear 2port stability using a single geometrically derived parameter. IEEE Transactions on Microwave Theory and Techniques, 1992, vol. 40, no. 12, p. 2303-2311. ISSN 0018-9480. DOI: 10.1109/22.179894

[17] EDWARDS, M. L., CHENG, S., SINSKY, J.H. A deterministic approach for designing conditionally stable amplifiers. IEEE Transactions on Microwave Theory and Techniques, 1995, vol. 43, no. 7, p. 1567-1575. ISSN 0018-9480. DOI: 10.1109/22.392916

[18] KUROKAWA, K. Some basic characteristics of broadband negative resistance oscillator circuits. The Bell System Technical Journal, 1969, vol. 48, no. 6, p. 1937-1955. ISSN 0005-8580. DOI: $10.1002 / \mathrm{j} .1538-7305.1969 . t b 01158 . \mathrm{x}$

[19] UGARTE-MUNOZ, E., HRABAR, S., SEGOVIA-VARGAS, D., et al. Stability of non-foster reactive elements for use in active metamaterials and antennas. IEEE Transactions on Antennas and Propagation, 2012, vol. 60, no. 7, p. 3490-3494. ISSN 0018926X. DOI: 10.1109/TAP.2012.2196957

[20] PlATZKER, A., STRUBle, W., HETZLER, K.T. Instabilities diagnosis and the role of $\mathrm{K}$ in microwave circuits. In IEEE MTT-S International Microwave Symposium Digest. Atlanta (GA, USA), 1993, vol. 3, p. 1185-1188. DOI: 10.1109/MWSYM.1993.277082 
[21] PLATZKER, A., STRUBLE, W. Rigorous determination of the stability of linear N-node circuits from network determinants and the appropriate role of the stability factor $\mathrm{K}$ of their reduced twoports. In The IEEE $3^{\text {rd }}$ International Workshop on Integrated Nonlinear Microwave and Millimeterwave Circuits. Duisburg (Germany), 1994, p. 93-107. DOI: 10.1109/INMMC.1994.512515

[22] OHTOMO, M. Stability analysis and numerical simulation of multidevice amplifiers. IEEE Transactions on Microwave Theory and Techniques, 1993, vol. 41, no. 6, p. 983-991. ISSN 00189480. DOI: $10.1109 / 22.238513$

[23] NYQUIST, H. Regeneration theory. The Bell System Technical Journal, 1932, vol. 11 , no. 1, p. 126-147. ISSN 0005-8580. DOI: 10.1002/j.1538-7305.1932.tb02344.x

[24] RANDALL, M., HOCK, T. General oscillator characterization using linear open-loop S-parameters. IEEE Transactions on Microwave Theory and Techniques, 2001, vol. 49, no. 6 , p. 1094-1100. ISSN 0018-9480. DOI: 10.1109/22.925496

[25] JACKSON, R.W. Criteria for the onset of oscillation in microwave circuits. IEEE Transactions on Microwave Theory and Techniques, 1992, vol. 40, no. 3, p. 566-569. ISSN 0018-9480. DOI: $10.1109 / 22.121734$

[26] STEARNS, S.D. Incorrect stability criteria for non-foster circuits. In Proceedings of IEEE 2012 International Symposium on Antennas and Propagation. Chicago (IL, USA), 2012, p. 1-2. DOI: 10.1109/APS.2012.6348832

[27] GONZALEZ-POSADAS, V., SEGOVIA-VARGAS, D., JIMÉNEZ, J.L., et al. Study of the stability properties of negative impedance converters using the gain-loop method. In IEEE Antennas and Propagation Society International Symposium (APSURSI). Washington (USA), 2011.

[28] KOLEV, S., DELACRESSONNIERE, B., GAUTIER, J.-L. Using a negative capacitance to increase the tuning range of a varactor diode in MMIC technology. IEEE Transactions on Microwave Theory and Techniques, 2001, vol. 49, no. 12, p. 2425-2430. DOI: $10.1109 / 22.971631$

[29] ALBARRACIN-VARGAS, F. Sensitivity analysis for active matched antennas with non-foster elements. IEEE Transactions on Antennas and Propagation, 2014, vol. 62, no. 12, p. 6040-6048. ISSN 0018-926X. DOI: 10.1109/TAP.2014.2364811

[30] HRABAR, S., KROIS, I., KIRICENKO, A. Towards active dispersion-less ENZ metamaterial for cloaking applications. Metamaterials, 2010, vol. 4, no. 2-3, p. 89-97. ISSN 1873-1988. DOI: $10.1016 /$ j.metmat.2010.07.001

\section{About the Authors ...}

Daniel SEGOVIA-VARGAS (M'98) was born in Madrid, Spain, in 1968. He received the telecommunications engineering and the Ph.D. degrees from the Polytechnic University of Madrid, Madrid, in 1993 and 1998, respectively. From 1993 to 1998, he was an Assistant Professor at Valladolid University, Valladolid. Since 1998, he has been an Associate Professor at Carlos III University, Madrid, where he is in charge of the microwaves and antenna courses. He has authored and coauthored more than 80 technical conference, letters, and journal papers. His research areas are printed antennas, active radiators and arrays, smart antennas, LH metamaterials, and passive circuits. He has also been member of the European Projects Cost260, Cost284, and COST IC0603.

José Luis JIMÉNEZ-MARTíN was born in Madrid, Spain, in 1967. He received the B.S. degree in Electrical
Engineering with a minor in radio-communication technical engineering, the M.S. degree in Telecommunications Engineering, and the Ph.D. degree from the Universidad Politécnica de Madrid (UPM), Madrid, in 1991, 2000, and 2005, respectively, and the master's degree in high strategic studies from CESEDEN (organization pertaining to the Spanish Ministry of Defense), Madrid, in 2007. He is currently an Associate Professor with UPM. He has authored or co-authored over 60 technical conference, letters, and journal papers. His current research interests include oscillators, amplifiers, and microwave technology.

Ángel PARRA-CERRADA was born in Madrid, Spain, in 1975. He received the B.S. and M.S. degrees in Electrical Engineering from the UPM, Madrid, in 1997 and 2003, respectively. He has been a Lecturer in Signal Theory and Communications with UPM since 2008. He is involved in the private sector in aeronautical and naval projects. His work is focused on electromagnetic interference and compatibility. His current research interests include secondary radio detection and ranging, active antennas, oscillators, microstrip antennas, composite right/left handed lines and metamaterials, microwave technology, and lightning protection.

Fernando ALBARRACIN-VARGAS was born in Bogotá, Colombia in 1985. He received the Engineer degree in Electronics from National University of Colombia, in 2008 and the Master in Multimedia and Communications from the Carlos III University in Madrid, Spain, in 2014. From 2010 to 2011, he was a Research Assistant with the International Centre for Physics in the Univesidad Nacional de Colombia, Bogotá, Colombia. He is currently working towards the Ph.D. degree with the Group of Radiofrequency, Electromagnetism, Microwaves and Antennas (GREMA) at the Carlos III University in Madrid. His present research interests include metamaterials based structures, non-Foster elements, ultra-wideband antennas, active-matching and miniaturization of antennas.

Eduardo UGARTE-MUÑOZ was born in Caracas, Venezuela in 1983. He received the Engineer degree in Telecommunications from the Carlos III University in Madrid, Spain, in 2008 and the Master in Multimedia and Communications in 2011. His research interests are metamaterials, active antennas, RFID systems, array antennas, metasurfaces, CMOS, non-Foster elements and active matching.

Vicente GONZALEZ-POSADAS was born in Madrid (Spain) in 1968. He received the Ing. Técnico in Radiocommunication Engineering degree (BS) in 1992, M.S. degree in Physics in 1995, and Ph.D degree in Telecommunication Engineering in 2001; Master degree in high strategic studies from CESEDEN (organization pertaining to the Spanish Ministry of Defense), Madrid, Spain, in 2009. He is working as an Assistant Professor at the Technical Telecommunication School, Polytechnic University of Madrid. His interests are related to active antennas, microstrip antennas, CRLH lines and metamaterials, microwave technology and RFID. He has authored or coauthored over 60 technical conference contributions, letters and journal papers. 